\title{
Neoadjuvant Chemotherapy (NACT) in Advanced Epithelial Ovarian Cancer: International Data and TMH Experience.
}

\author{
SUDEEP GUPTA
}

NACT followed by interval cytoreductive surgery has long been used in patients with advanced Stage III/IV ovarian cancer in most parts of the world other than North America. This was despite the fact that upfront surgery to optimal levels has been a dogma in this disease and there was lack of level 1 evidence to support NACT. The principal reasons for the usage of NACT have been the following:

1. Difficulty in performing optimal cytoreductive surgery in patients with extensive peritoneal disease. The required surgery in such patients is often so extensive that it is beyond the capabilities of all but few centres/ surgeons.

2. Difficulty in scheduling early surgery because of logistic reasons in many hospitals.

3. The poor nutritional status of many patients with advanced stage disease with presence of anemia, hypoalbuminemia etc. This results in prolonged convalescence after surgery with higher chance of morbidity and mortality.

There have been a large number of restrospective case series and reviews of NACT in ovarian cancer including systematic reviews based on these series. Some authors have also

Department of Medical Oncology, Tata Memorial Hospital

Mumbai-400012

E-mail: sudeepgupta@tatahospital.net proposed various CT scan and other criteria for selecting patients for NACT. However because of the retrospective nature of most of these reports and the lack of randomized controls there has been lingering doubt about this approach especially among the North American practitioners. However, given the fact that neoadjuvant chemotherapy has been non-inferior to upfront local treatment (and in some instances superior) in many other malignancies like breast, esophagus, gastric, urinary bladder etc. there has been a strong rationale to prospectively evaluate this approach in ovarian cancer.

Recently there have been reports from 2 randomized trials that have compared NACT followed by surgery to upfront surgery followed by adjuvant chemotherapy. One of them was conducted at AIIMS, New Delhi and will be further discussed by the lead investigator, Dr Kumar. The other trial was a combined effort of the European Organization for Research and Treatment of Cancer - Gynaecological Cancer Group (EORTC-GCG) and the National Cancer Institute Canada - Clinical Trials Group (NCICTG). The results of the Study were presented by the lead Investigator, Dr Ignace Vergote from University Hospital Leuven, Belgium, in the $12^{\text {th }}$ Biennial 12th Biennial Meeting of the International Gynecologic Cancer Society on October 25, 2008.

In this Study 718 patients with advanced stage IIIC or IV epithelial ovarian/peritoneal/fallopian carcinoma were randomized to receive either primary debulking surgery (followed by 6 cycles of platinum-based chemotherapy, which was 
mostly paclitaxel/carboplatin) or interval debulking surgery (which was preceded and followed by 3 cycles of chemotherapy). The study was powered as a non-inferiority study to prove that NACT was non-inferior to upfront surgery rather than as superiority design that would have required only 400 patients. The median follow-up for all participants was 4.8 years.

For the primary end-points of the Study there was no difference between the upfront surgery and NACT arms; median OS (29 Vs 30 months) and median PFS (12 Vs 12 months) being almost identical. For the secondary end points of the Study, which was the incidence of complications, the NACT arm fared better. Compared to the upfront surgery arm there were fewer post operative deaths $(2.7 \%$ Vs $6 \%)$, fever ( $2 \%$ Vs $8 \%)$, hemorrhage $(1 \%$ Vs $7 \%)$ and thrombosis $(0.3 \%$ Vs $2.4 \%)$ in the NACT arm. All of these were statistically significant. The rate of optimal debulking was also better in the NACT arm $(53 \%$ nil residual, $82 \%<1 \mathrm{~cm})$ compared to the upfront surgery $(21 \%$ nil residual, $46 \%<1 \mathrm{~cm}$ ).

In a multivariate analysis of the entire dataset by the investigators, optimal debulking surgery was the strongest independent prognostic factor for overall survival among the study participants in both groups $(\mathrm{P}=.0001)$. Other significant prognostic factors included: histological type $(\mathrm{P}=.0003)$, largest tumour size at randomization $(\mathrm{P}=.0008)$ and disease stage (IIIC Vs IV) $(\mathrm{P}=.0008)$.

The results of this Study provide support for the use of NACT/interval surgery in advanced ovarian cancer, but do not detract from the need for optimal surgery since this was still the strongest predictor of outcome.

At Tata Memorial Hospital about $70-80 \%$ of patients present in the advanced stages. The majority of these are offered NACT followed by surgery. The usual regimen in such patients is paclitaxel plus carboplatin. However after the publication of the ICON-3 trial, ${ }^{1}$ which showed the equivalence of single agent carboplatin to paclitaxel-carboplatin and CAP as adjuvant therapy, there was interest in our centre in evaluating single agent carboplatin as a neoadjuvant regimen.

\section{Methods and Results:}

From $31^{\text {st }}$ Oct. 2002 to 30 th April 2004, 46 consecutive patients of advanced EOC were enrolled. Thirty one patients $(67.4 \%)$ had stage 3 and 10 patients $(21.7 \%)$ had stage 4 disease. The indications for NACT included one or more of the following: massive ascites-32 (69.6\%), too extensive for optimal cytoreduction-12 (26.1\%), poor performance status-8 (17.4\%) and stage 4 disease-10(21.7\%).

All patients received NACT with carboplatin dosed to AUC 6 for a median of 3 cycles (range 3 to 4 ). There was a greater than $50 \%$ reduction or normalization of CA-125 (serological CR or $\mathrm{PR})$ in 42 patients $(91.3 \%)$. There was radiological $\mathrm{CR}$ or $\mathrm{PR}$ by standard WHO criteria in 24 patients $(52.2 \%)$. Thirty six patients were able to undergo subsequent surgery. In 21 of these 36 patients ( $45.6 \%$ of all patients) optimal cytoreduction could be achieved. The median intra-operative blood loss was $500 \mathrm{ml}$; median hospital stay was 6 days and there were no major intra-operative or post-operative complications. Pathological CR was observed in 2 patients $(4.3 \%)$. Grade $3 / 4$ neutropenia was observed in 2 patients $(4.3 \%)$, grade $3 / 4$ thrombocytopenia was observed in 5 patients $(10.8 \%)$ and grade $3 / 4$ anaemia developed in 13 patients $(28.2 \%)$. There were no episodes of febrile neutropenia or bleeding. Thirty four patients have subsequently received further adjuvant chemotherapy; single agent carboplatin was used in 23 patients and combination platinum therapy (paclitaxel-carboplatin or CAP) was used in 11 patients. Twenty six patients achieved complete remission at the end of all 
planned therapy of whom 8 have relapsed at the time of analysis. At a median follow-up of 8.3 months, the median event-free survival is 12.3 months. There have not been enough events to report overall survival.

\section{Conclusions:}

We concluded that single agent carboplatin has significant anti-tumour activity, comparable to standard combination regimens when it is used as neoadjuvant chemotherapy in advanced epithelial ovarian cancer. It is appropriate treatment when combination regimens cannot be used for any reason. It deserves to be studied in larger studies in this setting.

\section{REFERENCES:}

1. The International Collaborative Ovarian Neoplasm (ICON) Group. Paclitaxel plus carboplatin versus standard chemotherapy with either single-agent carboplatin or cyclophosphamide, doxorubicin, and cisplatin in women with ovarian cancer: the ICON3 randomised trial. Lancet 360:505-515, 2002. 